\title{
Tumor glômico: um diagnóstico fácil ou difícil?
}

\author{
Glomus tumor: an easy or a difficult diagnosis?
}

Gisela del Pilar Puentes

Buendia $^{1}$

William Albeiro JimeneZ

URIBE $^{1}$

Diogo Facciolli ${ }^{1}$

Juan Manuel Florez

RODRIGUEZ ${ }^{1}$

Jose De Gervais ${ }^{2}$
Trabalho realizado na Santa Casa de Misericórdia de Rio de Janeiro, Rio de Janeiro, RJ, Brasil.

Artigo submetido pelo SGP (Sistema de Gestão de Publicações) da RBCP.

Artigo recebido: 18/5/2010 Artigo aceito: 26/7/2010

\section{RESUMO}

Introdução: O tumor glômico é uma lesão neoplásica benigna, geralmente única, que se forma no glomo terminal. Esta estrutura é uma anastomose arteriovenosa subcutânea distribuída por toda a superfície corporal, em particular nos leitos ungueais, polpas digitais, palmas das mãos e plantas dos pés. Apesar da sua raridade, recebe atenção especial por seus sintomas caracterizados por dor paroxística, sensibilidade à pressão do local, ao frio e por sua localização típica na ponta do dedo. O diagnóstico inicial é raramente feito, levando os pacientes a apresentarem os sintomas por meses e até anos, sem o diagnóstico e tratamento adequados. Objetivo: O objetivo desse trabalho é evidenciar o método diagnóstico clínico e histopatológico do tumor glômico, e a utilização da microscopia em seu tratamento cirúrgico. Resultados: Observou-se grande diminuição nos casos de recidiva, com retorno funcional do órgão acometido e melhora da saúde física, funcional e psicossocial do paciente. Conclusão: A exérese do tumor mostrou-se eficaz e os resultados foram plenamente satisfatórios, não havendo complicações pós-operatórias significativas, nem recidivas até o momento.

Descritores: Tumor glômico/cirurgia. Neoplasias de tecido vascular. Doenças da unha/ cirurgia.

\section{SUMMARY}

Introduction: The glomus tumor is rare subungueal pathology that consists of a neuromyoarterial. Glomus bodies sensible to temperature oscillations, that regulate arteriolar flow, located at the arteriolar-venula anastomosis. The main symptom of this pathology is an excruciating pain leading to functional limitation of the Lyme. Objective: The purpose this study is to highlight its clinical and histopathological diagnosis, as well as the use of microcopy on its surgical treatment. Results: The treatment resulted in a great reduction of recurrence, with functional recovery of the limb and improvement of apaticutp's physical, social and psycosocial health. Conclusion: The surgical resection of the tumor was efficient and the results were very satisfactory, with no significant post operative complications and no recurrence.

Descriptors: Glomus tumor/surgery. Neoplasms, vascular tissue. Nail diseases/surgery.

1. Membro Residente do Serviço de Cirurgia Plástica e Cirurgia da Mão da Enfermaria $11^{\text {a }}$ da Santa Casa de Misericórdia do Rio de Janeiro.

2. Membro Titular da Sociedade Brasileira de Cirurgia Plástica; Regente do Serviço de Cirurgia Plástica e Cirurgia da Mão da Enfermaria $11^{\text {a }}$ da Santa Casa de Misericórdia do Rio de Janeiro. 


\section{INTRODUÇÃO}

O tumor glômico é uma lesão benigna que se desenvolve a partir de uma estrutura neuromioarterial existente na pele, o glomo, cuja função é regular a circulação sanguínea e a temperatura corpórea, constituído por uma arteríola aferente, um vaso anastomótico chamado canal de Soucquet-Hoyer, envolto por fibras musculares lisas, uma veia eferente, fibras nervosas e cápsula periférica ${ }^{1,2}$. A localização mais comum desse tumor é na mão, particularmente na região subungueal; no entanto, tem sido descrito em outros locais, porém $65 \%$ são localizados embaixo das unhas e, às vezes, bilaterais. $\mathrm{O}$ tumor glômico ou glomangioma pode também ser localizado no pênis, no pescoço e na orelha.

Sua primeira descrição foi realizada por William Wood, em 1812, descrevendo-o como um tumor subcutâneo intensamente doloroso, de crescimento lento e suscetível a variações de temperatura. Kolaczek, em 1878, observou uma lesão subungueal, sendo interpretado como um angiossarcoma. Em 1901, Grosser descreveu anastomoses arteriovenosas que se pensava estarem relacionadas com a regulação da temperatura. Os sinais e sintomas e a necessidade da excisão tumoral foi descrita por Barre, em 1920, que em 1924 se juntou a Pierre Mason para o estudo histopatológico desta enfermidade.

O objetivo deste trabalho é evidenciar o exame clínico e histopatológico como principal método diagnóstico, e também o uso da microscopia cirúrgica como um recurso para evitar a recidiva desta incapacitante e dolorosa doença.

\section{MÉTODO}

No período de janeiro de 2007 a novembro de 2009, 21 pacientes foram atendidos, examinados e submetidos à cura cirúrgica com auxílio da microscopia em nosso Serviço de Cirurgia Plástica e Reparadora da Mão da $11^{\mathrm{a}}$ Enfermaria da Santa Casa de Misericórdia do Rio de Janeiro.

A idade variou de 25 a 53 anos, sendo $19(90,47 \%)$ mulheres e $2(9,53 \%)$ homens. Todos relataram queixa álgica lancinante aos pequenos traumas e à exposição ao frio, assim sendo submetidos aos seguintes testes diagnósticos ao exame clínico:

- Ectoscopia e exame de transluminação ungueal-85,71\% apresentaram nódulo de coloração azul-violácea ou rósea no leito subungueal;

- Teste de Love ou Pintest - 100\% demonstraram sintomatologia dolorosa à percussão da lâmina ungueal com a cabeça de um alfinete na topografia tumoral;

- Teste de Hildreth - $(90,47 \%)$ relataram diminuição ou ausência do estímulo álgico à insuflação do esfignomanômetro a $300 \mathrm{mmHg}$, que leva à isquemia do membro acometido;

- Teste de sensibilidade térmica - por meio do estímulo com gelo ou água fria.

Outro exame que poderia ser utilizado, porém não realizado, seria a exposição ao spray de cloreto de etila, estimulando a dor local.

Todos os pacientes foram tratados cirurgicamente, por meio do bloqueio do plexo braquial, esvaziamento venoso e colocação de manguito pneumático com o dobro da pressão sistólica, incisão previamente demarcada em janela na lâmina ungueal (Figura 1). Realizado descolamento da lâmina ungueal de seu leito com auxílio da teta cânula (Figura 2), seguido de localização dos limites da tumoração por meio de microscopia e excisão da tumoração, respeitando seus limites de segurança até o periósteo da falange distal, e de curetagem desta (Figura 3). Foram realizados eletrocauterização da área excisada, curativo subungueal com colagenase, acomodação e fixação com fio de mononylon 4.0 da lâmina ungueal (Figura 4). Por fim, foi realizado curativo oclusivo e envio do material excisado para exame histopatológico.

Foram administrados $2 \mathrm{~g}$ de cefazolina per-operatória, permanecendo apenas analgesia pós-operatória. A fixação ungueal foi retirada após 14 dias de evolução.

\section{RESULTADOS}

Apenas dois dos 21 pacientes operados apresentaram complicações: um com recidiva da queixa álgica ao estímulo térmico, e outro evoluindo com granuloma subungueal, que regrediu espontaneamente com 35 dias de pós-operatório.

Os outros 19 pacientes retornaram a suas atividades diárias com reabilitação funcional do membro acometido.

Todos os resultados do exame histopatológico foram positivos para o tumor glômico.

\section{DISCUSSÃO}

O tumor glômico é lesão tumoral benigna, mais frequentemente localizado na polpa digital e região subungueal. A literatura também cita localizações mais raras, como sistema nervoso central, estômago, fígado, mediastino, traqueia, pulmões, ossos, articulações e órgãos genitais ${ }^{2-14}$. Corresponde a cerca de 1 a $5 \%$ dos tumores de mão. A incidência em relação a sexo e idade dos pacientes estudados em nosso trabalho está condizente com a literatura.

Histopatologicamente, o tumor está envolto por cápsula fibrosa, com numerosos espaços vasculares circundados por células endoteliais aplanadas e algumas camadas de células glômicas. Estas são de citoplasma eosinófilo, com grandes 


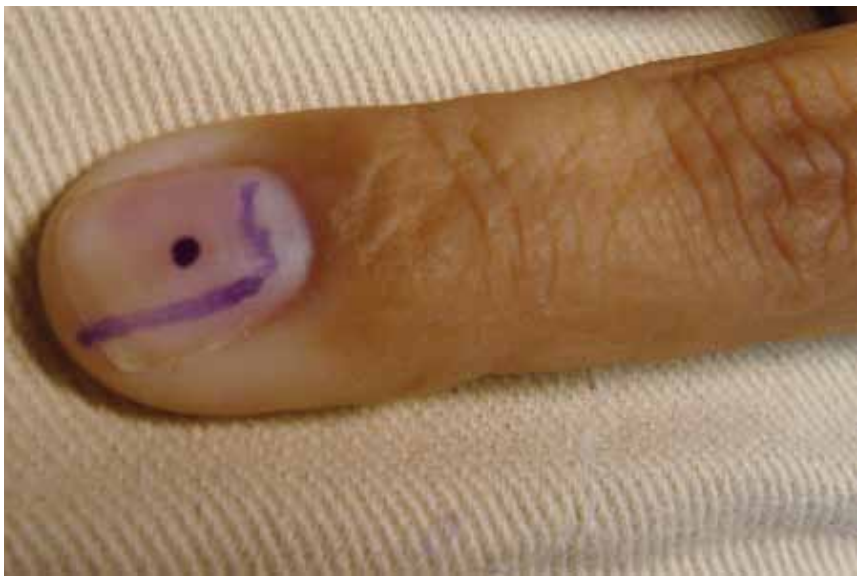

Figura 1 - Marcação em janela na lâmina ungueal.

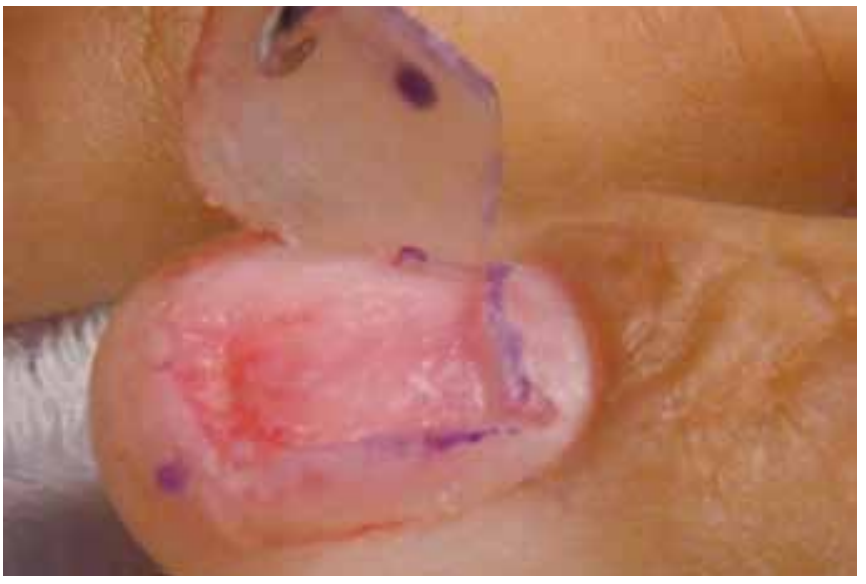

Figura 2 - Descolamento da lâmina ungueal de seu leito.

núcleos ovais ou cubóides, que se assemelham a células epiteliais ${ }^{4}$.

$\mathrm{O}$ aspecto do tumor é bastante característico; quando superficial, manifesta-se por um pequeno nódulo elástico e firme; a pele neste nível tem coloração roxo-escura ou púrpura. Se o tumor for mais profundo, apresenta-se endurecido e com estas mesmas tonalidades ${ }^{6}$. Clinicamente, caracteriza-se por hiperestesia e dor, geralmente bem localizada e que se acentua quando há exposição ao frio. A tríade de dor, sensibilidade ao frio e dor aguda à palpação faz pensar no diagnóstico de tumor glômico, o qual é caracterizado por sua sintomatologia hiperálgica.

O diagnóstico pode ser confirmado pelo estudo radiográfico, ressonância magnética e método de transiluminação. $\mathrm{O}$ exame radiológico da mão revela apenas erosão óssea, nos casos mais tardios. A ultrassonografia detecta apenas lesões maiores que $3 \mathrm{~mm}$ na região subungueal, não permitindo

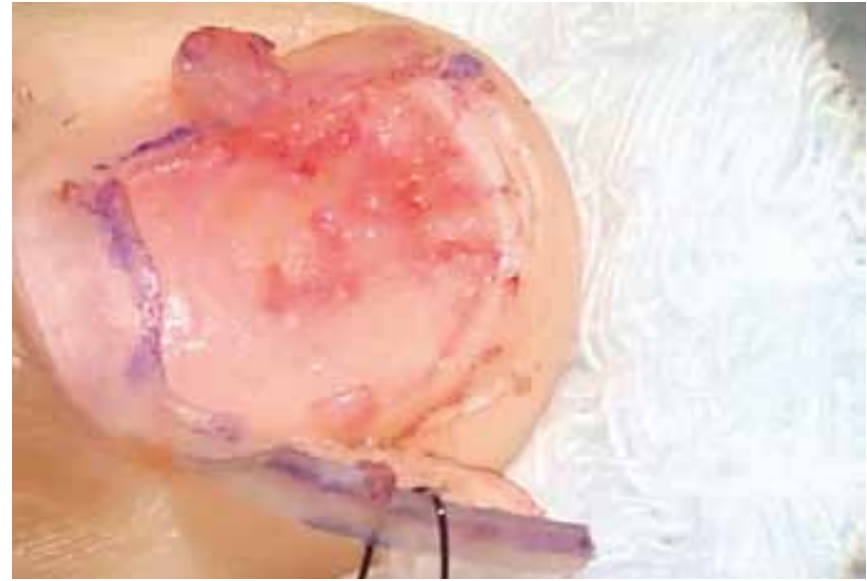

Figura 3 - Localização dos limites da tumoração por meio de microscopia e excisão da tumoração.

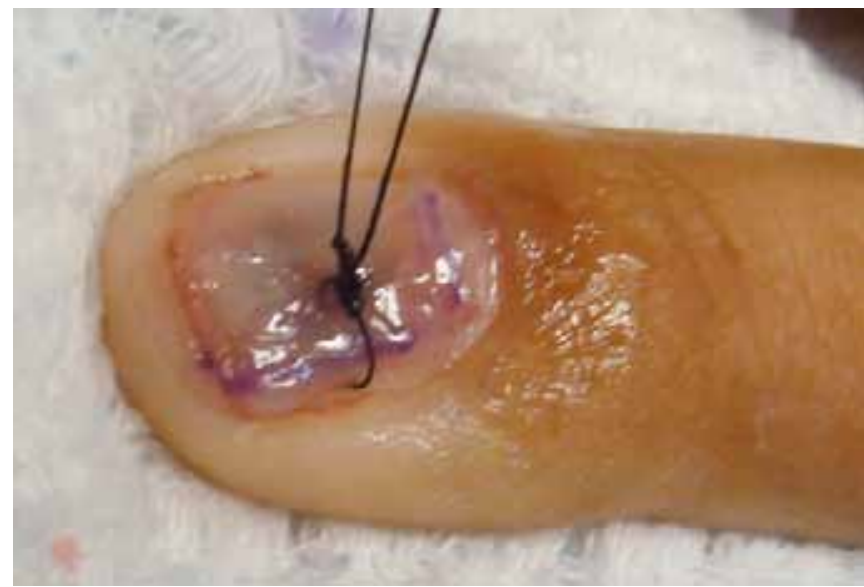

Figura 4 - Colocação e fixação da lâmina ungueal.

distinguir a natureza da lesão, além de ser examinador dependente. A ressonância nuclear magnética só detecta imagens nítidas, necessita de bobinas próprias e auxílio de profissional altamente especializado. A tomografia computadorizada e a angiografia são exames que também podem ser utilizados, porém também apresentando baixa sensibilidade para este tumor.

O diagnóstico diferencial deve ser feito com o hemangiopericitoma, neuroma, schwanoma, gota, artrose, melanoma subungeal e paroniquia crônica.

Basicamente, o tratamento desta lesão é feito pela excisão cirúrgica da mesma, sendo que a maior complicação é a recidiva ${ }^{4}$.

As complicações pós-operatórias descritas em literatura especializada incluem distrofia simpático-reflexa, infecção e sequela ungueal. A técnica cirúrgica utilizada com o uso de microscópio, a não ressecção da unha, sutura da matriz ungueal 
com fio absorvível e agulha atraumática tem como objetivo minimizar as complicações pós-operatórias, como defeito ungueal e cicatrização exuberante. A utilização de pequena cureta e cuidadosa cauterização do leito tumoral com bisturi elétrico bipolar diminuem as probabilidades de recidiva.

\section{CONCLUSÃO}

O tumor glômico é uma afecção rara, de diagnóstico clínico, sem a necessidade de exames complementares.

Três são os sintomas que vão nos sugerir o diagnóstico: dor espontânea lancinante irradiada ao antebraço e ombro, com ataques repentinos de hiperalgesia, dor provocada pelo toque, muitas vezes levando o paciente a evitar o mínimo toque ou carícia. Após estabelecimento de relação de confiança com o paciente, pode-se fazer o exame com um clips, permitindo uma localização precisa. Apresenta intolerância à mudança de temperatura, principalmente pelo frio; às vezes, a dor pode ser aliviada ao mergulhar as mãos em água morna. A combinação das três características é considerada patognomônico. O diagnóstico infelizmente é tardio, após a realização de múltiplos tratamentos sem resultados.

$\mathrm{O}$ tratamento cirúrgico microscópico desse tumor tornase um procedimento eficaz, reduzindo o número de recidivas, trazendo-nos um bom prognóstico para esta patologia limitante para o paciente.

\section{REFERÊNCIAS}

1. Bozza NG, Lanza O, Leza R, Abulafia J. Diagnóstico angiográfico de los tumores glómicos. Rev Argent Dermatol. 1981;62:117-23.

2. Taube MBP, Salles MJS, Carbonari MF, Vergnanini AL, Kato MVT. Tumor glômico solitário de localização rara: diagnóstico diferencial com síndrome de Parsonage-Turner. Rev Bras Reumatol. 1984;24:223-5.

3. Porto CC. Exame físico geral. In: Porto CC, ed. Semiologia médica. $4^{\mathrm{a}}$ ed. Rio de Janeiro:Guanabara Koogan;2001. p.111.

4. Souza CP, Aramaki O, Galbiatti JA, Durigan Jr A, Mizobuchi RR, Morais Filho DC, et al. Tumor glômico. Retrospectiva de nove casos: do diagnóstico ao tratamento. Rev Bras Ortop. 2000;35(6):214-8.

5. Hiraoka SR, Cauchioli CA. Tumor glômico da mão: apresentação de 19 casos e revisão da literatura. Rev Bras Ortop.1998;33(3):175-80.

6. Nazerani S, Motamedi MH, Keramati MR. Diagnosis and management of glomus tumors of the hand. Tech Hand Up Extrem Surg. 2010;14(1):8-13.

7. Schoen FJ. Vasos sanguíneos. In: Robbins patologia estrutural e funcional. $5^{\text {a }}$ ed. Rio de Janeiro:Guanabara Koogan;1996. p.449.

8. Lister GD, Pederson WC. Skin flaps. In: Green DP, ed. Green's operative hand surgery. $4^{\text {th }}$ ed. Vol. 2. New York:Churchil Linvingstone; 1999. p.2184-205

9. Boyes JH. Tumors. In: Bunnells surgery of the hand. $5^{\text {th }}$ ed. Philadelphia:Lippincott;1970. p.666-710.

10. Phalen GS, Mouroulis DL. The diagnosis and treatment of glomus tumor. Cleve Clin Q. 1966;33(2):73-9.

11. Camirand P, Giroux JM. Subungual glomus tumor. Radiological manifestations. Arch Dermatol. 1970;102(6):677-9.

12. Cernea SS. Experiência do grupo de cirurgia micrográfica de Mohs do HCFMUSP dez 1989 a abril de 1993. An Bras Dermatol.1994;69:365-73.

13. Cornell SJ. Multiple glomus tumours in one digit. Hand. 1981;13(3):301-2.

14. Gama C, França LC. Nerve compression by pacinian corpuscles. J Hand Surg. 1980;5(3):207-10.
Correspondência para:
Gisela Del Pilar Puentes Buendia

Rua do Russel, 344 - apto 605 - Rio de Janeiro, RJ, Brasil - CEP 22210-010

E-mail: gispuentesb@hotmail.com 